\section{TEORÍA DE CONJUNTOS Y ONTOLOGÍA}

\author{
Leticia 0. Minhot \\ Universidad Nacional de Córdoba (Argentina)
}

\section{SET THEORY AND ONTOLOGY}

\begin{abstract}
The question "what kind of entity is a scientific theory" is an ontological one. Its answer has to have ontological categories in order to submit theories. If these categories belong to set theory, then scientific theories are set theoretical entities. In this paper I develop three possible ways to explicate the claim that set theory is the ontology for scientific theories. The first one understands set theory as formal ontology in the sense of Husserl. The second one is based on Tugendhat (2003). Here set theory is interpreted as formal semantics. The third one has no relation to structuralist view of theories, but it shares with it the idea that set theory is ontology. This last way belongs to Badiou. The idea in this paper is to think the reconstruction of theories using set theory from a different way to the ontosemantic thesis.
\end{abstract}

KEY WORDS: Set theory; formal ontology; formal semantics; ontological decision.

\begin{abstract}
RESUMEN: Preguntarse qué clase de entidad es una teoría científica es plantearse una pregunta ontológica. Su respuesta consistirá de categorias ontológicas que subsumirán a las teorías. Si tales son las de la teoría de conjuntos, esto significa que las teorías científicas son entidades conjuntistas, al menos en parte. En este trabajo construyo tres modos posibles de elucidar la pretensión de la teoria de conjuntos como ontología para las teorías científicas. La primera es una lectura de la teoría de conjuntos como ontología formal en el sentido de Husserl. En la segunda, en base a Tugendhat (2003), el uso de la teoría de conjuntos para la reconstrucción de teorías significa que ésta es una semántica formal. La tercera es la presentación de una lectura ajena al estructuralismo, pero que, sin embargo, considera a la teoría de conjuntos como ontología. Lo que hago aquí es utilizar la propuesta de Badiou para pensar, desde una vía alternativa a la tesis ontosemántica, la actividad de reconstruir teorias utilizando a la teoria de conjuntos.
\end{abstract}

PALABRAS CLAVE: Teoría de conjuntos; ontología formal; semántica formal; decisión ontológica.

El elogio hecho por Lautréamont de las "matemáticas severas" es muy acertado. Lo que tienen de severas no es tanto el formalismo, o el encadenamiento de las demostraciones, como el hecho de que dejan al descubierto una máxima de pensamiento que podríamos formular del siguiente modo: justo en el momento en que decides lo que existe estás anudando tu pensamiento al ser. Pero entonces te encuentras, de manera inconsciente, sujeto al imperativo de una orientación.

Alain Badiou

Preguntarse qué clase de entidad es una teoría científica es plantearse una pregunta ontológica. Su respuesta debe ser, por lo tanto, una respuesta ontológica. Ésta consistirá de ciertas categorías ontológicas que subsumirán a las teorías (cfr. Moulines, 1982, 331). Si tales categorías son las de la teoría de conjuntos, esto significa que las teorías científicas son entidades conjuntistas, al menos en parte ${ }^{1}$. En este trabajo construyo tres modos posibles de elucidar la pretensión de la teoría de conjuntos como ontología para las teorías científicas. Desde estas tres variaciones procuro comprender el alcance de interpretar mediante formalización. La primera es una lectura de la teoría de conjuntos como ontología formal en el sentido de Husserl. Desde este juego hermenéutico, "interpretar formalizando" significa reconstruir basándose en una teoría formal del objeto. En la segunda, en base a Tugendhat (2003), el uso de la teoría de conjuntos como instrumento formal para la reconstrucción de teorías significa que ésta es, en tal uso, 
una semántica formal. Mientras estas dos lecturas guardan una relación de aproximación con la tesis ontosemántica de C.U. Moulines, la tercera se aparta de ella. Ésta es la presentación de una lectura ajena al estructuralismo, pero que, sin embargo, considera a la teoría de conjuntos como ontología. Lo que hago aquí es utilizar la propuesta de Badiou para pensar, desde una vía alternativa a la tesis ontosemántica, la actividad de reconstruir teorias utilizando a la teoría de conjuntos.

Al ser teorias científicas lo que estas categorias ontológicas permiten subsumir y al ser las primeras productos efectivos que podemos identificar en diferentes momentos de la historia de la ciencia o de la historia de las ideas, la teoria de conjuntos se transforma en un instrumento para la narrativa de dicha historia. ¿Qué significa que disponemos de tal instrumento para llevar a cabo tal relato? Cada uno de estos juegos interpretativos que aqui propongo aproxima una respuesta a esta pregunta.

\section{Ulises Moulines en su "Esbozo de ontoepistemoseman-} tica" (1998) sostiene lo siguiente:

Llamaremos "básicos" sólo aquellos términos que denotan los dominios empíricos de la teoria, su "ontología propia", o bien los que denotan espacios numéricos; los primeros, a su vez los podemos denominar "básicos principales", mientras que a los segundos podemos caracterizarlos como "básicos auxiliares". Llegamos pues a la conclusión de que en toda teoria hay que considerar al menos dos categorias lógicas de términos: básicos principales y relacionales. En la mayoría de teorias de las ciencias avanzadas tendremos dos categorias adicionales: los términos básicos auxiliares y los métricofuncionales. La primera pregunta central de ontoepistemosemántica que debemos plantearnos en este punto es si estas cuatro categorias lógicas de términos corresponden también a cuatro categorías ontológicas fundamentales de "seres". Esta problemática no puede plantearse en un vacío teórico, ni puede dejarse a la "intuición pura", sea eso lo que sea. Hay que asumir una teoría lo más general posible a modo de categorización ontológica básica -la que permite tratar "el Ser" del modo más general y menos comprometido posible. La teoría que aquí asumiremos como esquema ontológico general es la teoría elemental de conjuntos, o dicho con más precisión, la teoría fijada por la porción elemental del sistema Zermelo-Fraenkel (Moulines, 1998, 148, la cursiva me pertenece).
¿Qué significa que la teoría elemental de conjuntos es un esquema ontológico general? Esta teoría es el instrumento principal que utiliza la corriente llamada estructuralismo metacientífico -concepción que fue inaugurada por Sneed (1971) y desarrollada por Balzer, Moulines \& Sneed (1987)para la reconstrucción de teorias científicas efectivamente dadas en algún momento histórico. En principio, la teoria elemental de conjuntos no cumple funciones normativas respecto de las prácticas científicas, sino más bien interpretativas. Para Moulines, ésta es, justamente, la tarea de la filosofía de la ciencia, por medio de la cual codifica y sistematiza el discurso científico. El instrumento formal, la teoria de conjuntos, en este caso, tiene este objetivo: relatar de modo preciso y claro la historia de las ideas científicas. Es algo más que una herramienta al servicio del discurso histórico, es el discurso histórico mismo. Un discurso que se centra, según lo permite el instrumento, en los aspectos morfológicos de tales ideas. En el proyecto estructuralista metacientífico interpretar es matematizar, esto es, formalizar. Lo que se entienda por "formalizar" variará en cada una de estas alternativas.

La primera presentación que haré considera que comprometerse con la teoría de conjuntos como un esquema ontológico general significa que la teoría de conjuntos actúa como una ontología formal. Y entiendo por ontología formal lo que entendía Husserl, sólo que aqui se trata de una teoría matemática la que constituye esta disciplina. La ontología fue tradicionalmente una ocupación de los filósofos, por lo que aquí tendríamos una teoría matemática haciendo el trabajo de la filosofía. Husserl le critica a Kant el hecho de desarrollar su concepto de ciencia siguiendo a las ciencias particulares. La filosofía debe partir de los fundamentos de las ciencias, se trata de una investigación que debe buscar algo universalmente válido, esto es, la esencia de las cosas entendida como unidad de sentido. Sin embargo, considero que, a pesar de ello, entender la expresión "esquema ontológico general" al modo de "ontología formal" en el sentido de Husserl es una buena aproximación. El término "ontología", a lo largo de la historia de la filosofía, se ha referido a dos disciplinas diferentes. Por un lado, la ontología se ha identificado con metafísica y es la ciencia de la existencia en sentido propio al tratar del ser en sí, último e irreductible. Por otro lado, la ontología fue concebida como una teoría formal del objeto, es decir, como una teoría a priori de los objetos. Según este segundo sentido, la ontología sería una ciencia de las esencias -de 
lo que puede existir- y no de existencias -ciencia de lo que existe-. Así, una teoría formal del objeto es una teoría de lo que puede existir. Husserl, para quien la ontología se trata de una ciencia de esencias, no se ocupa del ente en cuanto tal, sino del objeto, y "esencia" es una unidad objetiva de sentido ideal. El autor distingue entre ontología formal y ontologías regionales o materiales. La primera trata de la determinación del ser y las segundas, las regionales o materiales, de las determinaciones que corresponden a cada tipo de ser. La realidad empírica es repartida en dominios diferentes en las ciencias empíricas. Pero todas y cada una de éstas manipulan conceptos en las cuales se fundan. Éstos se relacionan mutuamente y determinan estructuras necesarias en cada una de las regiones del ser. Cada parte de la realidad es una región que posee categorias propias cuyo dominio se circunscribe a esa ontología regional. Cada ciencia se ocupa de un ámbito específico de objetos. La concepción estructuralista considera a las teorias científicas como las unidades epistémicas que determinan lo que existe:

[...] el "compromiso ontológico" del que hablan Quine y sus seguidores tendrá, de preferencia, su lugar dentro del marco de una teoría; lo que realmente hay, depende de lo que las teorias realmente existentes (y aceptadas) admitan como entes concebibles (Moulines 1998, 143).

Los dominios empíricos de una teoría constituyen su "ontología propia" (cfr. Moulines 1998, 148). Sin embargo, según Husserl, los conceptos que caracterizan a cada parte de la realidad no son tematizados por la ciencia específica, sino que lo serán por una filosofía de la física, una filosofía de la química, etc. Las ontologías regionales son las filosofías de las ciencias particulares que tematizan y organizan los respectivos dominios de objetos, los cuales son concebidos como ámbitos disjuntos. La diferencia entre las regiones es una diferencia entre tipos de objetos. Además de esas categorías regionales hay conceptos comunes a todas las regiones de la realidad: categorías como objeto, relación, propiedad, etc. Se trata de categorias ontológicas fundamentales. Todas las ciencias las suponen y son tratados en la ontología formal. Ésta nos permite hablar del objeto independientemente de sus ámbitos respectivos. Ahora bien, el objeto que estudia la ontología formal no es el resultado de una abstracción desde la multiplicidad de objetos dados en las diferentes regiones. Se trata, más bien, de una formalización a priori del objeto en tanto objeto.
Las categorias formales, fundamento de toda posibilidad y de toda objetividad, subsumen a las categorias regionales, pero no deductivamente, sino que le dicen las formas de los objetos posibles. Y eso es formalizar en el sentido de Husserl. Las categorias formales no afirman nada acerca de las regiones que encuadran en su estructura universal ni establecen una esfera de la realidad por sí mismas.

Lo que tratamos de ver aquí es la teoría elemental de conjuntos funcionando como una ontología formal en el sentido en que Husserl entendía a esta disciplina. Pero, ¿qué significa que disponemos de una ontología formal para relatar la historia de las ideas? La teoria de conjuntos expondría el invariante de toda variación posible y su posibilidad. Una teoría empírica es posible en tanto pueda probarse que es una entidad conjuntista. La teoría de conjuntos como teoría del objeto evitaría el relativismo lingüístico en el que pudieran estar insertas las teorías, lo suprime por medio de una teoría que nos garantiza la comprensión de cualquier teoría científica. El ideal de claridad y objetividad es logrado por medio de la presentación del canon de objeto posible. Los objetos aquí son las teorias científicas. La "esencia" de tal objeto, es decir, la unidad objetiva de sentido ideal radica, en parte, en el carácter conjuntista de las teorías. Las teorias son entidades conjuntistas. Mi primera hermenéutica de "la teoría de conjuntos como esquema ontológico general" me inclina a pensar que Moulines está pensando esta teoría como una teoría del objeto, es decir, como una ontología formal, semejante en algunos puntos a como la pensara Husserl. Sin embargo, hay que hacer la siguiente salvedad: la teoría de conjuntos describe sólo una parte del objeto, ya que el elemento pragmático no se puede subsumir bajo tales categorias ontológicas.

Ahora bien, interpretar los aspectos morfológicos de teorías que se dieron efectivamente en un tiempo histórico desde una teoría formal a priori, implica transformar objetos que acaecieron históricamente en objetos ahistóricos. Sus aspectos morfológicos, al menos, se resuelven ahistóricamente en el canon de un modelo que nos regala la ilusión de liberarnos de toda perspectiva. La ontología formal trata como uniformes a los diferentes objetos históricos. Es el punto desde el cual podemos observar la variabilidad que presentan las diferentes teorías y sus modelos.

Mientras que esta lectura hizo énfasis en el "onto" de la tesis ontosemántica -puso al ser en situación de conjun- 
to- la que sigue lo hará en la parte "semántica". Para la segunda hermenéutica, la expresión "la teoría de conjuntos como esquema ontológico general" expresa que la teoría en cuestión funciona como una semántica formal. Podriamos argumentar a favor de esta hermenéutica que, para Moulines, la teoría de conjuntos utilizada como instrumento metateórico de las teorias científicas tiene como finalidad estudiar la estructura semántica de cualquier forma de teorización (Moulines, 1991, 67). Desde esta perspectiva, la teoria elemental de conjuntos, al funcionar como esquema ontológico general, estaría funcionando como una semántica formal. Esto es, funcionaría como la parte formal de una teoría de la referencia, pues la ontología estaría acorralada por el lenguaje y sólo se justificaría como aquello a lo que refiere el mismo, por las vías de los sentidos, al modo fregeano. Las categorias ontológicas fundamentales estarian en correspondencia con las categorías lógicas principales de las teorias.

Si creemos, como se desprende de MEM (Metodología Estructuralista Metacientífica) (y muchos otros enfoques reconstructivos del presente), que las unidades centrales para tratar la ontoepistemosemántica de la ciencia son los modelos de las teorias científicas, no tenemos más remedio que constatar que la elucidación más aceptable de la que disponemos acerca de lo que es un modelo es la debida a Tarski, y ella presupone el lenguaje de TEC (Teoria Elemental de Conjuntos). Mientras no dispongamos de una semántica formal alternativa igualmente potente (sea categorial, nominalista o lo que sea), debemos seguir admitiendo que un modelo de una teoría es una estructura en el sentido de TEC, compuesta por tanto de conjuntos y relaciones en el sentido de TEC (Moulines, 1998, 148-149).

La ontología formal, dijimos, tematiza al objeto en tanto objeto, esto es, sin considerar las regiones de objetividad. Para Husserl esta tematización se lleva a cabo mediante una formalización. Según Tugendhat, no queda muy claro en Husserl qué significa "formalizar" cuando se piensa ontológicamente. Ahora bien, "objeto", en un sentido amplio, es lo que puede ser sujeto de posibles predicaciones verdaderas. De allí, Tugendhat sostiene que la temática de la ontología formal se alcanza al reflexionar sobre el modo de nuestra manera de referirnos a los objetos (Tugendhat, 2003 , 44). En consecuencia, propone una ontología formal ampliada en una semántica formal. Husserl distinguía formalizar de abstraer, siendo la primera a priori y la segunda a posteriori. El problema de la abstracción o generalización radica en que, a medida que vamos subiendo el nivel de abstracción y logramos niveles más elevados, hay una negligencia de las diferencias, pues siempre se está buscando el elemento común que permita la generalización. De esta manera, vamos perdiendo a los objetos mismos y nos quedamos solamente con las categorias. El camino hacia niveles más elevados de abstracción no tiene retorno, es decir, no podemos, desde los conceptos, retornar a los objetos. ¿Cómo entender entonces la otra opción, es decir, la formalización? Para Tugendhat, la formalización objetual adquiere sentido en la formalización semántica (cfr. Tugendhat, 2003, 48). Cualquier consideración estructural de lo lingüístico es formal. De este modo, se puede diferenciar entre una semántica de contenido y una formal.

El especialista en la semántica de contenido puede preguntar por el significado de ésta y de otras expresiones. Si formalizamos lo que el científico (o cualquier otro usuario del lenguaje) hace cuando se refiere con este término singular a este objeto, si preguntamos qué significa en general referirse a un objeto y qué significa la noción de objeto ("qué 'es' un objeto en tanto que objeto"), esto lo hacemos de manera que formalizamos el planteamiento de contenido del semántico y preguntando por el significado formal de los términos singulares. [...] la ontología sólo se realiza en una filosofía analítica entendida como semántica formal (Tugendhat, 2003, 48-49).

Para Tugenthat la ontologia "se disuelve y se conserva por completo en la semántica formal" $y$, así, la teoría del objeto es, ahora, una parte de la semántica formal. Mientras la ontología formal se pregunta por el objeto en tanto que objeto, la semántica formal se pregunta qué significa comprender una frase. Esta idea no es tan ajena a la perspectiva ontoepistemosemántica de Moulines.

Basta admitir que hay tal componente no-referencial y que él es imprescindible en el análisis semántico de las teorías científicas como vía de acceso al referente y por tanto a la ontología en un contexto científico. [...] De aquí se desprende, una vez más, lo que ya hemos establecido desde el principio, a saber, que no pueden realmente separarse ontología, semántica y epistemología (Moulines 1998, 145).

Si bien, para la ontoepistemosemántica, la pregunta de la semántica formal trasciende a la comprensión de las fra- 
ses, dado que las unidades de significado son las teorias. Por lo que, la pregunta sería "¿qué significa comprender una teoría?". Sin embargo, teniendo esta precaución, voy a considerar, en esta hermenéutica, a la teoría elemental de conjuntos como una semántica formal, al modo en que Tugendhat lo entiende.

La ontología depende de la reflexión sobre las frases en las que hablamos de los objetos, es decir, de la semántica. Tugendhat demuestra la superioridad metodológica del análisis del lenguaje sobre la fenomenología en el estudio del concepto de conciencia de Husserl (cfr. Tugendhat, 2003,102 y ss).

Lo que demuestra es que toda conciencia intencional no proposicional implica una conciencia intencional proposicional. La conciencia de un objeto no es representar a ese objeto sino referirse a él. La referencia a un objeto por medio de un término singular forma parte de la comprensión de las frases predicativas. La referencia a un objeto reposa en el hecho de que consideramos verdadera a una frase existencial. De esta forma, Tugendhat muestra que "toda conciencia intencional es explícita o implícitamente conciencia proposicional" (Tugendhat, 2003, 111).

Lo que puede decirse es regido, dice Tugendhat, por la semántica formal. Todos los planteamientos decisivos de la ontología griega resultaron de una reflexión semántica, si bien era seguida de una interpretación que la transformaba en una reflexión sobre objetos ocultando su dimensión lingüística.

La teoría del objeto forma parte de la semántica formal. Tanto la pregunta "¿qué significa referirse a objetos?" como "¿qué significa la noción 'objeto'?" conducen a un planteo semántico formal. Así, la ciencia formal que está en la base de todas las ciencias es la semántica y no la teoría del objeto. Pero la condición formal de la semántica no se limita a los términos singulares, sino que se extiende a las frases. De este modo, para Tugendhat, la semántica formal no sólo subsume a las determinaciones predicativas, sino que alcanza a la teoría del objeto misma. Para la concepción estructuralista, las unidades de significado son las teorías y sus unidades más simples son los modelos, en el sentido que lo entiende la teoría formal de modelos. $Y$ los modelos de una teoría son estructuras en el sentido de la teoría elemental de conjuntos, de este modo, esta última, es una semántica formal que subsume a la teoría del objeto. Que la teoría elemental de conjuntos sea un esquema ontológico general significa que todo lo que hay está en los conjuntos que componen los modelos de una teoría. Desde esta lectura, cualquier discurso sobre los objetos que existen o sobre la conciencia conlleva siempre, de modo implícito o explícito, una reflexión semántica del significado formal de los términos y de las frases -en la semántica formal de Tugendhat-o de las teorías en donde se dice -en el estructuralismo metacientífico-. La semántica formal es, para Tugendhat, una ciencia formal universal. Una ciencia eminente. Para la concepción estructuralista, una metateoría.

Ahora, ¿qué significa que disponemos de una semántica formal para relatar la historia de las ideas? El discurso histórico está determinado, nuevamente, por un discurso formal, sólo que ahora discurre como semántica. Esto implica, una vez más, el problema de la ahistoricidad, el relato de lo histórico se hace desde un instrumento ahistórico. Las teorías, con sus aspectos morfológicos propios, son instancias de esta morfología general. La adopción de una semántica formal nos permite disponer de un patrón de sentido que subsume las semánticas de contenido propias de las teorías. "Interpretar formalizando" significa que podemos percibir los rasgos morfológicos comunes de las teorías.

[...] al ocuparnos metateóricamente de la física, obtenemos una determinada comprensión de la estructura semántica de cualquier forma de teoretización (Moulines, 1991, 67).

La tercera interpretación de la pretensión de hacer de la teoría elemental de conjuntos el esquema ontológico general para reconstruir las teorías científicas no intenta ser una lectura de la posición de Moulines. Lo que en este punto presento es una posibilidad más de lo que significa dicha pretensión. En esta tercera vía interpretativa, la teoría elemental de conjuntos es una ontología, no formal como era el caso de Husserl, sino ontología a secas, en el sentido en que Badiou lo entiende: una disciplina que trata de lo que hay de pensable antes de que haya objetos. Tradicionalmente, el discurso que se ocupaba del ser se inscribía en la filosofía; ahora, la única disciplina capaz de tal tratamiento es la matemática: cualquier tratado acerca del ser es un tratado matemático. Pero con esto no debe entenderse una tesis metafísica que enuncia que 
el ser esté compuesto de estructuras matemáticas. Es, al contrario, una identificación de discursos: el discurso sobre el ser, ontológico, es un discurso matemático. "Las matemáticas, en todo su devenir histórico, enuncian lo que puede decirse del ser-en-tanto-ser" (Badiou, 1999, 16). Según Badiou (2002), cuando la matemática, históricamente, debió reflexionar sobre sí misma, es porque estaba atravesando un periodo de "crisis". La crisis de los irracionales en la matemática pitagórica, la crisis de las paradojas en teoría de conjuntos, crisis relacionada con los elementos infinitamente pequeños en el siglo XVIII, etc. En estos momentos la matemática vuelve sobre sí misma por el surgimiento de una anomalía, un problema que le impide avanzar. Y debe tomar posición, esto es, debe tomar una decisión ontológica. Según el autor, todo pensamiento es polémico, no porque entren en conflicto diferentes interpretaciones, sino que el conflicto se da entre diferentes juicios de existencia.

El pensamiento de Husserl y el de Tugendhat es un pensamiento intencional, esto es, dirigido a objetos. El ser queda en Husserl delimitado a la esfera del objeto. El núcleo central de su filosofía intencional es la estructura de la conciencia respecto a un objeto. En Tugendhat, el foco de su propuesta es la estructura semántica del lenguaje con respecto a los objetos. En la tesis de Badiou, la ontología que se identifica con las matemáticas no es una tesis metafísica, porque no afirma que la realidad de las entidades matemáticas se da de modo independiente a todo sujeto de conocimiento. No postula entidades últimas e irreductibles. Tampoco es una teoría formal de los objetos ni una teoría de la referencia. Para Badiou, el discurso del ser-en-tanto-ser no presenta objetos como referentes del discurso matemático. $Y$, a su vez, la ontología no es el referente de lo lingüístico como pretende la perspectiva semántica de la ontología. Todo objeto es el resultado de una operación que supone una ley o estructura que le da unidad. Una estructura es la ley que transforma una multiplicidad pura en multiplicidad de unidades. Pero ese tipo de estructuras trabaja en los discursos de las teorías científicas, no en la ontología.

La decisión conjuntista de Badiou lo lleva a concebir la ontología como ciencia de lo múltiple en tanto múltiple. Lo múltiple no debe ser pensado aquí como multiplicidades de individuos, sino lo múltiple como previo a cualquier operación de estructura que genera unidades. Es una multiplici- dad sin individuos y por ello sólo puede ser matemática. Y aqui debemos elucidar la tarea de la formalización. No se trata de formalizar al objeto ni a una estructura lingüistica. La única estructura que un discurso ontológico admite es la axiomática, pues no contiene la ley que produce unidades. La formalización es la estructura del discurso ontológico en tanto tal y es inherente al discurso. Las ontologías propias de las teorias empiricas son multiplicidades en las que ya ha operado una estructura y ha producido unidades, por eso sus multiplicidades son multiplicidades de unidades. La ontología es un pensamiento axiomático que no expone objetos, sino lo pensable sin convertirlo en tema. Los términos de esta axiomática no tienen sentido por tener un referente, sino por estar en el "juego pautado de sus conexiones fundantes" (Badiou, 2002, 31). La formalización presenta el juego de las conexiones que prescribe la decisión ontológica y presenta el logos del ser previo a su objetivación. Previo no significa a priori, previo significa antes de que actúen las estructuras que generan objetos.

En el programa que propone Badiou se diluye la prioridad formal y lingüistica de lo gramatical sobre la decisión ontológica; a la inversa, la ontología determina el lenguaje y su gramática. Con "formalización" no se entiende aquí una gramática universal, porque toda investigación ontológica es local. Esto significa que el ser siempre se presenta bajo las constricciones de una exposición local. La decisión ontológica decide el discurso. La formalización, en tanto estructura inmanente del discurso, es inherente a la decisión ontológica. Una teoría es matemática si mediante una decisión determina una existencia y su consistencia es determinada exclusivamente por esa decisión. Mientras la lógica piensa los mundos posibles, la ontología decide un mundo. Y esta decisión se lleva a cabo sin que ningún algoritmo externo la determine. La lógica está sometida a la localidad de la decisión ontológica. Un mundo matemático localiza su propia lógica, pues fija correlaciones constrictivas entre sus características ontológicas y su lógica inmanente. La lógica se subordina a la ontología, y por ello puede ser matematizada (cfr. Badiou, 2002, 116).

Lo que hasta ahora he presentado son tres modos de concebir la ontología. La de Husserl, donde la ontología formal responde a una voluntad de fijar exteriormente un marco ontológico formal a las ontologías regionales. Estas últimas están oprimidas por las normas que la primera les impone respecto de las estructuras de los objetos. Por 
su parte, Tugendhat propone una ontología reducida a la semántica formal. También aquí existe la voluntad de fijar exteriormente un marco formal a la ontología, pero, a diferencia de Husserl, no es ontológico sino semántico. La semántica formal funciona como una gramática que rige a la ontología. Tanto la ontología formal de Husserl como la semántica formal de Tugendhat tratan de lo posible racional. De este modo conllevan una interpretación de lo racional $y$, en este sentido, son evaluativas para esa interpretación de racionalidad. Son el patrón que otorga uniformidad a las variaciones que presentan las teorías. Funcionan como protocolos que controlan la racionalidad. Tanto una como otra son separables de lo efectivo. En el caso de Badiou, la ontología no es teoría del objeto sino de la multiplicidad pura, es decir, multiplicidad sin los principios que determinen sus unidades. La ontología expone, sin definir ni nombrar, lo que hay de pensable en la multiplicidad pura. De este modo, la ontología presenta lo múltiple sin convertirlo en objeto. La localidad del pensamiento ontológico es la prueba de la ausencia de la voluntad de fijar exteriormente un protocolo formal válido para todo universo posible. Por lo que, a diferencia de Husserl, no hay una ontología formal y ontologías regionales, no hay una oposición entre lo formal y lo empírico, hay una pluralidad de multiplicidades cuyas presentaciones tienen cohesiones inmanentes. La esencia de la matemática no es la formalización entendida como forma a priori del objeto o del lenguaje, sino que consiste en ser ontología. La matemática no establece normas externas que sirvan para evaluar universos matemáticos posibles, sino que la matemática decide un universo. $Y$ cuando decide un universo fija la lógica inmanente del mismo. Decidir un universo es una decisión que le permite al pensamiento acceder a lo que él mismo declara como existente.

Ahora bien, si admitimos la tesis de Badiou, ¿qué significa que reescribimos la historia de las teorías científicas utilizando la teoría de conjuntos? La respuesta es que pensamos la historia de las ideas desde una decisión ontológica. Hacemos universal lo local. Hacemos presente el pasado. Bueno, después de todo, sólo podemos captar el pasado y lograr comprenderlo desde los lentes que nos ofrece el presente. La homogeneización de las teorías que le brinda el aparato formal son los lentes desde los cuales se miran otras formas, otros estilos. La teoría de conjuntos no es ni una gramática ni una teoría del objeto, la teoría de conjuntos es una decisión ontológica que conlleva una formalización que le es propia. Se trata de proyectar el significado que "hay" tiene en un universo a todos los universos. Apelar a la teoría de conjuntos como tesis ontológica es sostener que la multiplicidad es axiomáticamente homogénea (cfr. Badiou, 2002, 53). Esto implica que las complejidades de los aspectos morfológicos de las teorías son aprehendidos de un modo fijo y homogeneizados bajo un plan que coloca a las diferentes teorías científicas que han aparecido en distintos momentos históricos bajo una lógica de equivalencia, al menos en lo que se refiere a sus morfologías estructurales.

En sintesis, con Husserl tenemos ontología como teoría del objeto, en Tugendhat, como teoría de la referencia, y, en Badiou, como teoría de la multiplicidad pura al tomar la decisión conjuntista. Deseo terminar este trabajo con algunas reflexiones sobre la adopción de esta ontología. La homogeneidad no es, en definitiva, más que otro nombre para la ahistoricidad. Lo que tienen en común las tres opciones es que no se busca una aprehensión móvil de las complejidades morfológicas presentes en los productos que emergen a lo largo de la historia de la ciencia. La actividad reconstructiva se lleva a cabo desde un esquema que en sí mismo opera ahistóricamente. Si bien la teoría de conjuntos no subsume a toda la teoría, pues el elemento pragmático es externo al aspecto formalizable, cabría preguntarse si este elemento introduce el elemento histórico. Pero eso es tema para otro trabajo; me he abocado aqui a la parte que cae bajo la teoría de conjuntos. Y los aspectos así presentados lo son de un modo ahistórico. La historicidad que aquí se refiere no es la de una reducción de todas las teorías científicas a otra teoría dada en algún momento histórico. No se reduce, por ejemplo, la física de Newton a la de Einstein. La ahistoricidad a la que me refiero es a nivel de metalenguaje. Tenemos así un dispositivo fijo: las teorías científicas son presentadas como casos de ese dispositivo. Las teorías son objetos históricos, que se dieron efectivamente en un espacio y en un tiempo determinados. Pero la comprensión a la que accedemos formalizando es ahistórica. A esta objeción puede responderse que toda comprensión de tales objetos es siempre desde un punto, de una mirada que tiñe todo lo que toca. La teoría elemental de conjuntos es, en este caso, tal mirada. 


\section{NOTA}

1 En el programa de Sneed se adopta el método de Suppes de identificar las teorias empíricas mediante predicados conjuntistas, pero se introducen otros elementos. La identidad de la teoría no depende sólo del predicado conjuntista sino también del dominio de las aplicaciones propuestas. De este modo, la respuesta ontológica, además del predicado conjuntista, introduce un elemento externo al mismo. En la delimitación de tal dominio entran en juego consideraciones que no pertenecen a la teoría de conjuntos (cfr. Moulines, 1982, 342 y ss.).

\section{BIBLIOGRAFÍA}

Recibido: 23 de junio de 2009 Aceptado: 10 de enero de 2010
Badiou, Alain (2002): Breve tratado de ontología transitoria, Barelona: Gedisa.

Balzer, Wolfgang, C. Ulises Moulines y Joseph D. Sneed (1987): An Architectonic for Science, Dordrecht: Reidel.

Moulines, C. Ulises (1982): Exploraciones metacientíficas. Estructura, desarrollo y contenido de la ciencia, Madrid: Alianza.

Moulines, C. Ulises (1991): Pluralidad y recursión. Estudios epistemológicos, Madrid: Alianza.

Moulines, C. Ulises (1998): "Esbozo de ontoepistemosemántica", Theoria 13 (31): 141-159.

Sneed, Joseph D. (1971): The Logical Structure of Mathematical Physics, Reidel: Dordrecht.

Tugendhat, Ernst (2003): Introducción a la filosofía analítica, Barcelona: Gedisa. 